Check for updates

Cite this: Phys. Chem. Chem. Phys., 2020, 22, 23553

Received 16th June 2020

Accepted 16th September 2020

DOI: $10.1039 / \mathrm{d} 0 \mathrm{cp} 03228 \mathrm{j}$

rsc.li/pccp

\title{
A statistical thermodynamics view of electron density polarisation: application to chemical selectivity
}

\author{
Frédéric Guégan, (D) *a Vincent Tognetti, (D) b Jorge I. Martínez-Araya, \\ Henry Chermette, (D) d Lynda Merzoud, ${ }^{d}$ Alejandro Toro-Labbée and \\ Christophe Morell (iD *d
}

\begin{abstract}
A fundamental link between conceptual density functional theory and statistical thermodynamics is herein drawn, showing that intermolecular electrostatic interactions can be understood in terms of effective work and heat exchange. From a more detailed analysis of the heat exchange in a perturbation theory framework, an associated entropy can be subsequently derived, which appears to be a suitable descriptor for the local polarisability of the electron density. A general rule of thumb is evidenced: the more the perturbation can be spread, both through space and among the excited states, the larger the heat exchange and entropy.
\end{abstract}

\section{Introduction}

From the very beginning, density functional theory (DFT) and thermodynamics have been closely connected. ${ }^{1}$ This analogy very likely started with the treatment of a set of electrons as an inhomogeneous gas by Hohenberg and Kohn. ${ }^{2}$ It has been later reinforced by the development of a Gibbs-Duhem equation counterpart. ${ }^{3}$ The coining of the Lagrange multiplier associated with the energy minimization after the thermodynamics chemical potential pursued even further the connection. ${ }^{4}$

Many macroscopic concepts have also found their analog in DFT. The definition of pressure ${ }^{5}$ and temperature $e^{6-8}$ of an electron system and the proposal of a maximum entropy principle ${ }^{9,10}$ are other examples. ${ }^{11}$ More recently, temperaturedependent conceptual DFT descriptors ${ }^{12-16}$ and temperaturedependent charge transfers have been paid a lot of attention, even though it has become quite clear that the temperatures required for tangible electron density modifications are much

\footnotetext{
${ }^{a}$ IC2MP UMR 7285, Université de Poitiers - CNRS, 4, rue Michel Brunet TSA, 51106-86073 Cedex 9 Poitiers, France. E-mail: frederic.guegan@univ-poitiers.fr

${ }^{b}$ Normandy Univ., COBRA UMR 6014 - FR 3038, Université de Rouen, INSA Rouen, CNRS, 1 rue Tesniére, 76821 Mont St Aignan, Cedex, France

${ }^{c}$ Departamento de Ciencias Quimicas, Facultad de Ciencias Exactas, Universidad Andres Bello (UNAB), Av. República 498, Santiago, Chile

${ }^{d}$ Université de Lyon, Institut des Sciences Analytiques, UMR 5280, CNRS, Université Lyon 1 - 5, rue de la Doua, F-69100 Villeurbanne, France.

E-mail: christophe.morell@univ-lyon1.fr

${ }^{e}$ Laboratorio de Química Teórica Computacional (QTC), Facultad de Química,

Pontificia Universidad Católica de Chile, Santiago 7820436, Chile
}

larger than the macroscopic temperatures used in routine experimental chemistry, ultimately suggesting that "electronic" and thermodynamic temperatures are two separate quantities.

It will be shown in this article that the interaction with an external perturbation may modify the electron density in such a way that it would be equivalent to a change in a well-defined electronic temperature. To prove it, we follow the traditional line by applying statistical physics concepts to DFT. Through this approach, the work and heat exchange between an electron system and its surroundings will be defined, and the definitions of the associated entropy and temperature will be subsequently provided. Interestingly, the temperatures calculated through this analogy are in agreement with the temperatures needed to make an electron density evolve.

Thus this paper is organized as follows. In the following section, we discuss some previous temperature and entropy definitions in conceptual theoretical chemistry, in order to unravel the differences between our approach and those already reported in the literature. In the third one, basic relations between statistical physics and thermodynamics are recalled. The fourth section then exploits the reminded concepts to devise the analogy with conceptual $\mathrm{DFT}^{17-19}$ and to define electronic work and heat counterparts, while section five more specifically deals with the concepts of entropy and temperature. Then, in section seven, these concepts are applied to a few molecular systems, such as acrolein, thiophene and pyrrole using the methodology outlined in section six. The article ends with some concluding remarks. 


\section{Temperature and entropy in theoretical chemistry}

The purpose of this section is to highlight (without aiming at being comprehensive) some peculiarities of our approach by confronting it with some other conceptual schemes dealing with temperature. Note already that the aim here is not to define an exact explicit temperature-dependent density functional theory, but rather to use temperature as a probe for some specific property of interest. Saying otherwise, our aim here is conceptual and not computational DFT. Briefly (and with a little oversimplification), with this premise, two main objectives can be delineated for the use of temperature in DFT.

The first one is mainly developing a computational tool targeted to improve or diagnose the methods. This is for instance the case of thermally-assisted-occupation $\mathrm{DFT}^{20,21}$ where a "fictitious" temperature is introduced in order to add flexibility for the design of accurate exchange-correlation functionals by involving virtual orbitals (VOs), while, in principle, the use of VOs is avoided in exact Kohn-Sham (KS) theory. Still within the KS framework, Grimme and Hansen considered finite-temperature DFT to characterise the multi-reference character of molecules and to assess the amount of static correlation. $^{22}$ This is also usual in DFT solid state calculations. ${ }^{23}$

The second goal is related to the rationalisation of chemical behaviours and in particular of reactivity. For instance, Scheffler and co-workers introduced the change of the electron density due to the excitation of low-energy electron-hole pairs induced by an increased electronic temperature as a quantity that characterises the spatial distribution of the reactivity of metal surfaces. ${ }^{24}$ It illustrates Chermette's statement that "local reactivity indices may be introduced, taking into account variations with respect to variables [...] such as the electron temperature, $T$, which induces electronic excitations". ${ }^{19}$

There were thus several proposals for electronic temperature and electronic entropy based on the electron density. Among them, we should cite the Ghosh-Berkowitz-Parr (GBP) definition, ${ }^{1,25}$ which reads:

$$
S_{G B P} \propto \int \rho(\mathbf{r})\left(c+\ln \frac{t_{s}(\mathbf{r})}{t_{T F}(\mathbf{r})}\right) \mathrm{d} \mathbf{r},
$$

where $t_{\mathrm{s}}$ and $t_{\mathrm{TF}}$ denote the KS fictitious and Thomas-Fermi kinetic energy densities, respectively. A local temperature was associated according to (with $k_{\mathrm{B}}$ being the Boltzmann constant):

$$
\beta(\mathbf{r})=\frac{1}{k_{\mathrm{B}} T(\mathbf{r})}=\frac{3 \rho(\mathbf{r})}{2 t_{\mathrm{s}}(\mathbf{r})} .
$$

Another relevant work is the extensive use of information theory by Liu, and in particular of the Shannon entropy and Kullback-Leibler divergence, to unravel chemical reactivity, to retrieve main electronic effects and to provide predictive models. ${ }^{17,26-28}$ The key formula of this approach is:

$$
S_{\mathrm{S}}=\int \rho(\mathbf{r}) \ln \rho(\mathbf{r}) \mathrm{d} \mathbf{r} .
$$

The works by Heidar-Zadeh, Ayers and Nalewajski ${ }^{29-31}$ on the axiomatization of atoms-in-molecules partitions (with applications to the study of bonding) ${ }^{32}$ based on such quantities also deserve to be mentioned. More fundamentally, as proved by Nagy, such defined entropy determines every property of a finite Coulomb system. ${ }^{33}$

Accordingly, all these works intend to extract physicochemical information from a given electron density. Our goal, here, is different: we do not aim to characterise the electron density by itself and as it is, but, rather, its propensity to be modified or distorted. Within the so-called canonical representation, two factors can make it evolve: either a variation of the electron number, or of the external potential.

Variations associated with the change of electron number have been the topic of many papers since the seminal PerdewParr-Levy-Balduz article. ${ }^{34}$ The prototypical case is that recently reviewed by Gázquez and collaborators: ${ }^{14}$ "the system under consideration is highly diluted in the solvent, which acts as an electron reservoir, so that the number of electrons in the system will fluctuate because it can exchange electrons with the solvent". In the so-called three-state model, the fractional number of electrons around $N_{0}$ is modelled by a mixing of the $N_{0}-1, N_{0}$, and $N_{0}+1$ states. Then, the average number of electrons can be expressed by (see also Chapter 4 in Parr and Yang's textbook ${ }^{35}$ ):

$$
\langle N\rangle=N_{0}+\frac{\mathrm{e}^{\frac{A+\mu}{k_{\mathrm{B}} T}}-\mathrm{e}^{-\frac{I+\mu}{k_{\mathrm{B}} T}}}{1+\mathrm{e}^{\frac{A+\mu}{k_{\mathrm{B}} T}}+\mathrm{e}^{-\frac{I+\mu}{k_{\mathrm{B}} T}},}
$$

where $A$ and $I$, respectively, denote the vertical electron affinity and first ionization potential of the considered system, and $\mu$ is the electronic chemical potential. In this grand-canonical ensemble, the associated entropy is then defined using the corresponding partition function $Z$ and the average energy $\langle E\rangle$ :

$$
S_{\mathrm{GC}}=k_{\mathrm{B}}\left(\ln Z+\frac{\langle E\rangle-\langle N\rangle \mu}{k_{\mathrm{B}} T}\right) .
$$

It it thus plain to see that this temperature $T$ here controls the average fraction of electrons exchanged with the surroundings. As a consequence, to quote Franco-Pérez, ${ }^{13}$ "it is now wellaccepted that the temperature that one uses when exploring chemical reactivity concepts is not the true temperature but an "effective" temperature that models in a qualitative way, interactions between reactants during a chemical reaction", and, even more precisely, it is the charge transfer component of such a reaction.

Additionally, electron density variations due to the external potential can be split into two categories: those induced by displacements of nuclei associated with the atoms of the molecular system itself, and those triggered by an additional potential that does not belong to the initial system (this is the case for instance when a reaction partner approaches). The first category is closely related to molecular vibrations. In such cases, the relevant temperature is that associated with the nuclei kinetic energy, which, by virtue of the equipartition theorem, is directly linked to the thermodynamical temperature defined in 
the $N V T$ ensemble. In a recent paper, ${ }^{36}$ molecular dynamics simulations (using classical nuclei) were performed to assess to what extent electron density-based reactivity descriptors are affected when structural breathing is described by thermal nuclei moves.

Here, we target the second category: the changes in the electron density associated with the addition of a new external potential, and we will show in Section 4 how a specific temperature can be associated with it. To this aim, some thermodynamical key concepts have to be preliminarly introduced.

\section{Statistical physics background}

It is textbook knowledge to retrieve thermodynamics from statistical physics. ${ }^{37}$ Through this approach, work, heat, entropy and temperature can be derived from probability distributions within the appropriate statistical ensemble.

Take a closed system that can exchange heat $(\delta Q)$ and work $(\delta W)$ with its surroundings. The first principle of thermodynamics, which merely translates the energy conservation of the system, states that:

$$
\mathrm{d} U=\delta W+\delta Q
$$

Within the canonical ensemble, the internal energy can be written as:

$$
U=\sum_{i} p_{i} E_{i}
$$

in which $p_{i}$ and $E_{i}$ are, respectively, the population and the energy of the $i$ th state. If one lets the system undergo a transformation in which heat and work are exchanged, the internal energy variation is given by:

$$
\mathrm{d} U=\sum_{i} \mathrm{~d} p_{i} E_{i}+\sum_{i} p_{i} \mathrm{~d} E_{i},
$$

where the first right hand side term corresponds to a change in the state populations, while their energies remain constant. On the contrary, the second term describes the variation of the energy levels at constant population.

Now let $\lambda$ be a parameter of the Hamiltonian describing the system. The associated force is:

$$
\hat{F}=\frac{\partial \hat{H}}{\partial \lambda} .
$$

Upon infinitesimal variations of $\lambda$, each energy level is modified according to the Hellmann-Feynman theorem:

$$
\mathrm{d} E_{i}=\left\langle\Psi_{i}|\hat{F}| \Psi_{i}\right\rangle \mathrm{d} \lambda=-\mathrm{d} W_{i},
$$

where $\mathrm{d} W_{i}$ is the work provided by the surroundings. Its statistical average is

$$
\delta W=\sum_{i}\left\langle\Psi_{i}|\hat{F}| \Psi_{i}\right\rangle \mathrm{d} \lambda p_{i}=\sum_{i} p_{i} \mathrm{~d} E_{i}
$$

Hence the work exchange is clearly ascribed to the variation of energy levels at constant population. Therefore, the second right hand side term of eqn (8) can be identified with the heat exchange. This last result can actually be derived independently. Indeed, the corresponding Gibbs-Shannon entropy is given by:

$$
S=-k_{\mathrm{B}} \sum_{i} p_{i} \ln p_{i}
$$

For closed systems, the number of particles is constant, $\sum \mathrm{d} p_{i}=0$. Hence,

$$
\mathrm{d} S=-k_{\mathrm{B}} \sum_{i} \mathrm{~d} p_{i} \ln p_{i}
$$

within the canonical (NVT) ensemble, probabilities are given by:

$$
p_{i}=\frac{1}{Z} \mathrm{e}^{-\beta\left(E_{i}-E_{0}\right)},
$$

with $Z$ the partition function:

$$
Z=\sum_{j} \mathrm{e}^{-\beta\left(E_{j}-E_{0}\right)},
$$

$E_{0}$ being the ground state energy taken as energy of origin, and $\beta=\left(k_{\mathrm{B}} T\right)^{-1}$. Hence,

$$
\begin{aligned}
\mathrm{d} S & =-k_{\mathrm{B}} \sum_{i} \mathrm{~d} p_{i}\left(\ln \mathrm{e}^{-\beta\left(E_{i}-E_{0}\right)}-\ln Z\right) \\
& =-k_{\mathrm{B}} \sum_{i} \mathrm{~d} p_{i}\left(-\beta\left(E_{i}-E_{0}\right)\right)-k_{\mathrm{B}} \ln Z \sum_{i} \mathrm{~d} p_{i},
\end{aligned}
$$

so that, still for a closed system,

$$
\mathrm{d} S=\frac{1}{T} \sum_{i} \mathrm{~d} p_{i}\left(E_{i}-E_{0}\right) .
$$

The associated heat is thus,

$$
\delta Q=T \mathrm{~d} S=\sum_{i} \mathrm{~d} p_{i}\left(E_{i}-E_{0}\right) .
$$

It is plain to see that, for a quantum system exchanging work and heat with its surroundings, on the one hand, the work exchange can be seen as a modification of the total energy due to a change in the state energy, while their populations remain unchanged. On the other hand, the heat exchange can be seen as an evolution of the total energy induced by a change in the state occupations.

We now apply these results to the fundamental equations of conceptual DFT.

\section{Work and heat in conceptual DFT}

For the sake of simplicity, one considers in the first stage a closed electron system that can only exchange energy with its surroundings. Systems able to exchange particles and therefore undergo chemical reactions will be the subject of another paper. The average electronic energy can be written as:

$$
E=\sum_{i} p_{i} E_{i}
$$

in which $p_{i}$ is the population of the electronic state of energy $E_{i}$, $E_{0}$ and $p_{0}$ being, respectively, the energy and the population of the ground state. It is well known that at room temperature 
only the electronic ground state is (at equilibrium) generally significantly populated. For instance, a temperature of $20000 \mathrm{~K}$ would be required to populate the first excited state of hydrogen by 1 percent of the total population. Therefore, the latter equation reduces to:

$$
E=E_{0}
$$

Let us now imagine that this system is perturbed by, for instance, an electrostatic external potential created by a point charge. The energy variation of the ground state is, up to the second order, given by:

$$
\mathrm{d} E=\int \rho(\mathbf{r}) \delta v(\mathbf{r}) \mathrm{d} r+\frac{1}{2} \iint \chi\left(\mathbf{r}, \mathbf{r}^{\prime}\right) \delta v(\mathbf{r}) \delta v\left(\mathbf{r}^{\prime}\right) \mathrm{d} \mathbf{r} \mathrm{d} \mathbf{r}^{\prime},
$$

$\delta \hat{v}=\hat{H}-\hat{H}_{0}$ being the perturbation Hamiltonian, $\rho(\mathbf{r})$ the electron density and $\chi\left(\mathbf{r}, \mathbf{r}^{\prime}\right)$ the so-called static linear response kernel. The first right hand side term of the latter equation is the linear Stark effect energy. It actually corresponds to the first order energy correction in perturbation theory:

$$
E^{(1)}=\left\langle\Psi_{0}\left|\left(\hat{H}-\hat{H}_{0}\right)\right| \psi_{0}\right\rangle,
$$

and also to the variation of the ground state energy at constant electronic configuration (since this first-order perturbation does not populate the other states):

$$
E^{(1)}=\left\langle\Psi_{0}\left|\left(\hat{H}-\hat{H}_{0}\right)\right| \psi_{0}\right\rangle=p_{0} \mathrm{~d} E_{0} .
$$

By analogy, one identifies this term to the work exchange. In the case of a negative point charge around the system, then $\delta v(\mathbf{r})>0$. The system is thus providing work to its surroundings.

$$
\delta W=\int \rho(\mathbf{r}) \delta v(\mathbf{r}) \mathrm{d} \mathbf{r}>0 .
$$

On the contrary, in the case of a positive point charge interacting with the system, then $\delta v(\mathbf{r})<0$ and $\delta W<0$, so that the system is receiving work from the medium.

In a recent paper by the same authors, ${ }^{38}$ it has been shown that the second right hand side term of eqn (21), the polarisation energy, can be seen as an electron reconfiguration energy describing how the electron density is adapting to the new external potential. The perturbed wave-function can then be projected upon the basis set formed by the unperturbed eigenvectors,

$$
\Psi_{\mathrm{P}}=\sum_{k} c_{k} \times \Psi_{k}
$$

The population of each excited level is $\mathrm{d} p_{k}=c_{k}^{2}$, the ground state being finally populated by $\mathrm{d} p_{0}=1-\sum c_{k}^{2}$. This polarisation energy can be expressed as:

$$
E^{(2)}=\frac{1}{2} \iint \chi\left(\mathbf{r}, \mathbf{r}^{\prime}\right) \delta v(\mathbf{r}) \delta v\left(\mathbf{r}^{\prime}\right) \mathrm{d} \mathbf{r} \mathrm{d} \mathbf{r}^{\prime}=-\sum_{k \neq 0} \mathrm{~d} p_{k} \times\left(E_{k}-E_{0}\right),
$$

where the population of the excited state $k$ reads:

$$
\mathrm{d} p_{k}=\left(\frac{\left\langle k\left|\hat{H}-\hat{H}_{0}\right| 0\right\rangle}{E_{0}-E_{k}}\right)^{2}=c_{k}^{2},
$$

and the corresponding electron density polarisation is given by

$$
\delta \rho(\mathbf{r})=\int \chi\left(\mathbf{r}, \mathbf{r}^{\prime}\right) \delta v\left(\mathbf{r}^{\prime}\right) \mathrm{d} \mathbf{r}^{\prime}=2 \sum_{k \neq 0} c_{k}\left\langle\Psi_{k}|\hat{\rho}(r)| \Psi_{0}\right\rangle .
$$

where $\hat{\rho}$ is the one-electron density operator. Thus, the second right-hand side term of eqn (8) can be identified with the heat exchange

$$
\delta Q=-\sum_{k \neq 0} \mathrm{~d} p_{k} \times\left(E_{k}-E_{0}\right)
$$

In summary:

$$
\mathrm{d} E=p_{0} \times \mathrm{d} E_{0}-\sum_{k} \mathrm{~d} p_{k} \times\left(E_{k}-E_{0}\right)=\delta W+\delta Q .
$$

It is quite clear that an electron system perturbed by a point charge mainly induces a work exchange between the system and the point charge. Indeed, the work is the first order correction to the total energy. This work is accompanied by a small exchange of heat due to the reshuffling of the electron density, which is actually a cooling of the system. Indeed, whatever the perturbation, the polarisation energy is always stabilizing. Interestingly, the cooling can be the main effect if the perturbation is due to a dipole. In this case, the work done by the positive and the negative ends of the dipole cancels out, and the polarisation/heat transfer takes over it. Besides, this cooling is associated with a modification of the energy level population. At this stage, it would thus be appealing to define the temperature of the perturbed system.

\section{Entropy and temperature}

The Gibbs-Shannon ${ }^{39}$ entropy of the unperturbed system is null (in analogy with Nernst's third law of thermodynamics) ${ }^{40}$ since only the ground state is populated $\left(p_{0}=1\right.$, and $p_{i}=0$ for $i \neq 0$ ) and thus

$$
S_{\text {unpert }}=-k_{\mathrm{B}} \sum_{k} p_{k} \ln p_{k}=-k_{\mathrm{B}}\left(p_{0} \ln p_{0}\right)=0 .
$$

Once the electron system is perturbed, a certain number of states $\left|\Psi_{k}\right\rangle$ of energy $E_{k}$ become populated by a fraction $\mathrm{d} p_{k}$ of the total electron number. The entropy variation is thus given by:

$$
\mathrm{d} S=-k_{\mathrm{B}} \sum_{k} \mathrm{~d} p_{k} \ln \mathrm{d} p_{k}
$$

The sum over the states includes the ground state, whose population is after perturbation $p_{0}+\mathrm{d} p_{0}=\left(1-\sum_{i \neq 0} \mathrm{~d} p_{i}\right), \mathrm{d} p_{i}$ being the population of the $i$ th excited state. $\mathrm{d} S$ actually translates the spreading of the electron density over the excited states. It can be seen as a measure of how dispersed the 
electronic polarisation is, both in real space and among the energy states. Thus, a polarisation that leads to a wide electron density reshuffling would exhibit a large entropy. We stress that only static polarisation is considered here, i.e. the case of entropy of electromagnetic polarisation, more tricky, ${ }^{41-43}$ is not approached in this work.

In Section 2, the connection between entropy, heat and temperature was done through the Boltzmann distribution in which the population of an excited state $k$ depends on the ratio between the energy difference and the temperature. Thus, when one raises the temperature, the lowest excited states are populated first. In the problem at hand, the probability of having the level $k$ populated is more intricate since it is given by:

$$
\mathrm{d} p_{k}=\left(\frac{\left\langle k\left|\mathcal{H}_{\text {pert }}\right| 0\right\rangle}{E_{0}-E_{k}}\right)^{2} \text {. }
$$

Should the numerator of the latter equation be the same for all the excited states, then a simple statistical distribution would be easily found. Indeed, the occupation would be a unique function of the inverse of excitation energies. Unfortunately, the numerator makes the connection between the ground state and the excited state through the perturbation potential. Therefore the numerator can be quite important for high excited states, and the population distribution may not be monotonic. Consequently, high excited states can be much more populated than lower ones. In this situation, a temperature can be hard to define rigorously. However, it will be shown in the next section that as an empirical relation exists between entropy and polarisation energy (heat), a temperature can be calculated. Indeed, one can use the macroscopic definition:

$$
T=\frac{\partial E}{\partial S}
$$

It has been previously mentioned that the electron density reshuffling induces a cooling of the system. The temperature is required to drop from the unperturbed system whose temperature is already $0 \mathrm{~K}$. In the current context, to get positive temperature values, the absolute value of the energy in eqn (34) was considered. This temperature translates the energy that would have been needed to distort the electron density, according to:

$$
T=\frac{\partial E}{\partial S}=\frac{\mathrm{d}\left(\left|\sum_{k}-\mathrm{d} p_{k} \times\left(E_{k}-E_{0}\right)\right|\right)}{\mathrm{d}\left(-k \sum_{k} \mathrm{~d} p_{k} \ln \left(\mathrm{d} p_{k}\right)\right)}
$$

Alternatively, one can conceive $T$ as the temperature of the electron system subjected to perturbation, so to say, "how hot the perturbed electron density is". For a specific molecule, one can compute for different locations of the perturbation the polarisation energy and the associated entropy, and then compute a numerical derivative that has physical features of a temperature.

It is easily seen that if the perturbation is due to a point charge, the electric charge that generates the electrostatic potential will increase the population of every state. In that regard, the electrostatic charge can be seen as a temperature counterpart. It indicates how "hot" the point charge is. The dependence of temperature on the small electric charge $\delta q$ of the point charge perturbation is not difficult to delineate. From eqn (33), the level occupations are proportional to $\delta q^{2}$. This leads to the following dependence for the temperature:

$$
T \propto \frac{1}{\ln \delta q^{2}}
$$

As a final remark for this section, let us notice that the gist of thermodynamics is essentially making averages. In the previous equation, a sum is made on all the considered perturbations of the external potential, which are independently imposed on the system. This can be compared with the other definitions presented in Section 2. Indeed, in eqn (1) and (3), the sum is made on all real space points where the electron density is evaluated. In eqn (5), the sum is carried out on the states featuring different electron number values. In the case where temperature controls the nucleus movements, the sum depends on the vibrational modes. This freedom on the choice of summation index accounts for the variety of possible temperature definitions in theoretical chemistry.

\section{Computational details}

All gas phase geometries and wavefunctions were obtained at the B3LYP/6-31+G* level of theory using ORCA 4.0 software. ${ }^{44}$ In all cases, the first 50 excited states were computed using the Tamm-Dancoff approximation (TDA). Transition densities for all 50 excited states were extracted in the cube file format using the orca_plot utility. They were used to evaluate the different descriptors analysed herein by a home made Fortran program that can be obtained from the authors upon request. Integration was performed by direct summation on the cube grids, whose spacing was in all cases less than 0.15 bohr. Potential perturbations were located at each nucleus of relevant atoms, which is in a way reminiscent of the $\mathrm{H}^{*}$ method. ${ }^{45,46}$

We would like to stress the fact that even if DFT was used here to generate all data, the post-Hartree-Fock (post-HF) methods could also have been used without any difficulty, since our approach only requires electron densities and transition densities that are in principle also available with the postHF calculations. In that sense, the use of the "conceptual DFT" phrase should not be seen as restricting the method application to the sole DFT.

\section{Polarisation entropy of selected molecular systems}

In this section, the concepts previously defined are applied to several representative molecular systems, namely propenal (aka acrolein), thiophene and pyrrole. 


\subsection{Acrolein}

Propenal, more widely known as acrolein, is an enal (conjugated carbonyl system).

It is well known that this molecule is prone to react with nucleophiles. The carbonyl carbon, hereafter numbered C3, reacts readily with "hard" (in the sense of Pearson's hard and soft acids and bases (HSAB) theory) ${ }^{47}$ nucleophiles. The conjugated carbon or $\beta$ carbon, numbered $\mathrm{C} 1$, reacts better with "soft" nucleophiles leading for instance to the so-called Michael additions, which are among the most widespread techniques to form carbon-carbon bonds. On the other hand, the oxygen atom of the carbonyl group generally undergoes complexation with metal cations or protonation. The first stage of a nucleophilic attack on acrolein is represented in Fig. 1.

Carbon 1, the conjugated carbon, is considered as the most polarisable atom and therefore as softer. Within the HSAB principle, this atom reacts best with soft bases. On the other hand, carbon 3 is considered as the least polarisable, or the hardest. It is prone to combine with hard bases. Another way to explain the reactivity of $\mathrm{C} 1$ is to look at the reaction intermediate. In Fig. 1 it appears that the attack on carbon 1 leads to the most stable intermediate because the additional charge is best spread among the molecule.

To assess the polarisability of each carbon, a perturbation has been successively set on their nucleus. In Fig. 2 the density polarisation maps for the acroleine perturbed on C1(A), C2(B) and on $\mathrm{C} 3(\mathrm{C})$ are represented.

The density accumulation regions are colored in red, while the density depletion regions are in yellow. The perturbation by a negative point charge of $q=-0.1 e$ successively located on each carbon induced a density depletion on the corresponding carbon. These density polarisation maps are a way to describe how the density is reshuffled or spread among the molecule, which atoms gain or lose electron density. The biggest reshuffling is reached when the perturbation is located on $\mathrm{C} 1$, which is consistent with the fact that it is the most polarisable amongst the carbon backbone. To get a more quantitative assessment, the polarisation energy and entropy have been calculated.

In Fig. 3, the polarisation spectrum for the 50 first excited states is represented along with the polarisation energy and the corresponding entropy for each carbon. The polarisation spectrum describes on the $Y$ axis the transition probability $\mathrm{d} p_{k}$ triggered by the perturbation with respect to the excited state

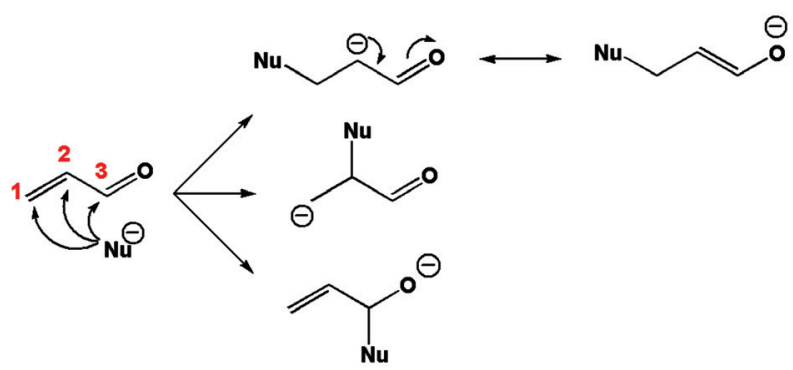

Fig. 1 First stage of the nucleophilic attack mechanism on acrolein.
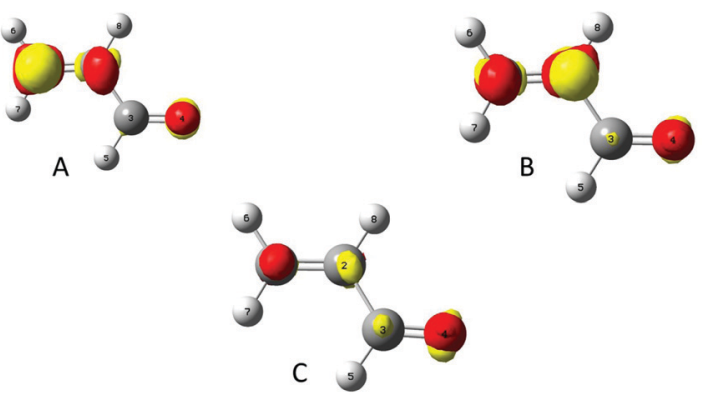

Fig. 2 A, B and C indicate that perturbation is applied, respectively, on the nucleus of atoms C1, C2 and C3. Density polarisation maps. isovalue of 0.0005 e bohr $^{-3}$.

number $k$ on the $X$ axis. The Gibbs-Shannon entropy has been computed for the three positions of the perturbation, namely on atoms C1, C2 and C3. It is plain to see that for carbon C1 fewer excited states are involved than those of the two other carbons. However the transition probabilities are about one order of magnitude higher. So even though the polarisation is distributed on fewer excited states, its magnitude is much higher. The entropy is therefore higher when the charge is located on $\mathrm{C} 1$ than on other carbons.

The polarisation energy, the second order correction to the energy, which is, according to Section 4, the heat exchange, was also calculated. The polarisation energy follows the same trend as the polarisation entropy. The polarisation energies are $-8.52 \times 10^{-3} \mathrm{eV},-5.81 \times 10^{-3} \mathrm{eV}$, and $-3.52 \times 10^{-3} \mathrm{eV}$ for carbons 1, 2 and 3, respectively. So, the bigger the entropy, the smaller the polarisation energy, and the larger the stabilization. As a consequence both the polarisation energy (heat exchange) and the polarisation entropy seem to be good candidates to indicate the most polarisable atom among the carbon backbone. Indeed, the results show a strong correlation between the polarisation energy and the polarisation entropy, which holds as far as our perturbative calculation remains valid.

Finally, it might be interesting to assess the temperature associated with the different reshuffling. Provided that it is applicable, eqn (34) indicates that temperature can here be expressed as the slope of the $E=f(S)$ plots. In Fig. 4, the

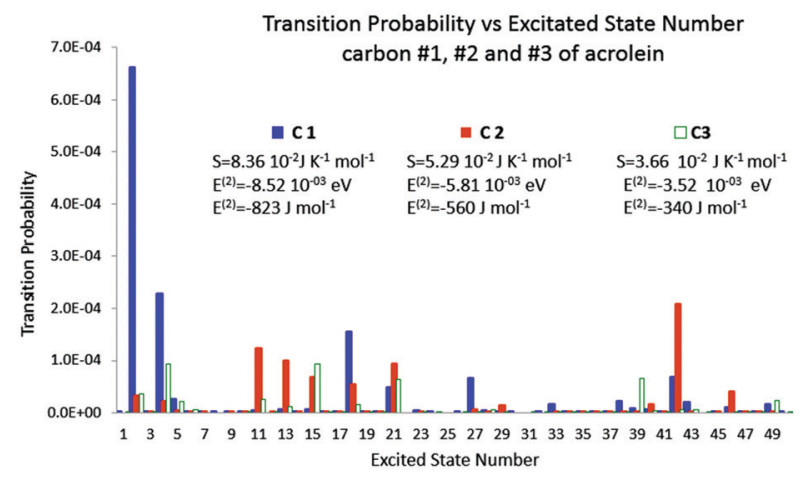

Fig. 3 Polarisation spectrum when $\mathrm{C} 1, \mathrm{C} 2$ and $\mathrm{C} 3$ are perturbed by a $-0.1 \mathrm{e}$ point charge. 


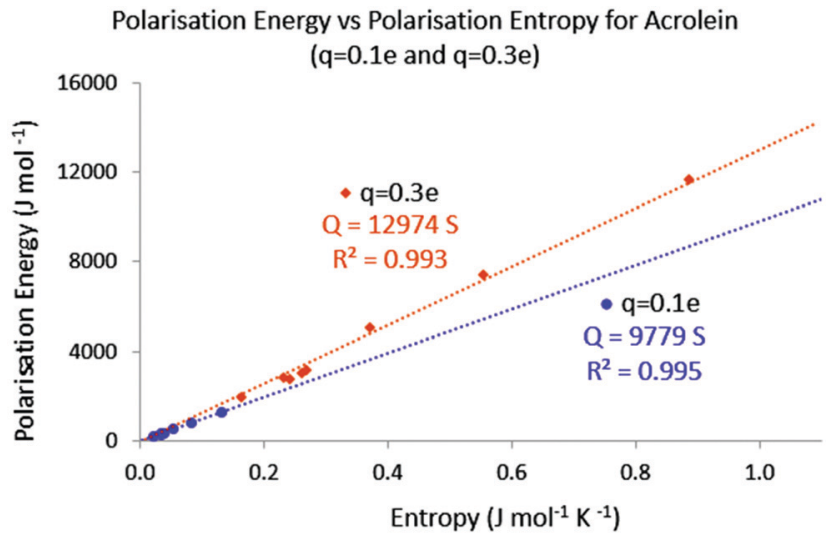

Fig. 4 Absolute polarisation energy versus entropy; $q=-0.1$ e and $q=$ -0.3 e.

absolute values of the polarisation energy with respect to the polarisation entropy for, respectively, a point charge perturbation of $q=-0.1 e$ and $q=-0.3 e$ applied on all the atoms of acrolein are represented. The $(E=0, S=0)$ point was also added to the data sets, as the unperturbed system is expected to display both null polarisation energy and entropy.

It is believed that the number of $E=f(S)$ points is large enough to draw some conclusions. In both cases the polarisation energy and the polarisation entropy are linearly connected. For a point charge perturbation of $q=-0.1 e$ located at the nucleus of each atom, the corresponding temperature (slope) is about $9800 \mathrm{~K}$. For a point charge perturbation of $q=-0.3 e$ the equivalent temperature is about $13000 \mathrm{~K}$. These levels of temperature are in agreement with the temperature needed to distribute 1 percent of the total population of a molecule collection on the first excited state. As noted in other temperature-based developments, and as stated earlier, this temperature is also much higher than the "macroscopic" temperature used in chemical synthesis. Interestingly, even though the heat transfer and polarisation entropy values change with the location of the point charge perturbation, the global temperature remains the same and seems only dependent on the charge magnitude.

To evaluate the temperature dependence on charge, we considered again nuclei-centred perturbations, the point charge magnitude varying from $-5 \times 10^{-4}$ to $-5 \times 10^{-1} e$. Linearity of the $S=f(E)$ plot is maintained in all cases $\left(R^{2}\right.$ being above 0.99 in all the cases). Moreover, the slope variation with the charge complies with the expected $1 / \ln q^{2}$ dependence, as shown in Fig. 5, thus reinforcing confidence in the found results.

\subsection{Thiophene and pyrrole}

Reactivities of thiophene and pyrrole are very comparable. They generally undergo aromatic electrophilic substitution at the $\alpha$ carbon, the carbon neighbour to the heteroatom. ${ }^{48}$ The limiting step of this reaction is the addition of the electrophile on the aromatic system, which is indeed highly endothermic and thus, according to Hammond's postulate ${ }^{49}$ is associated

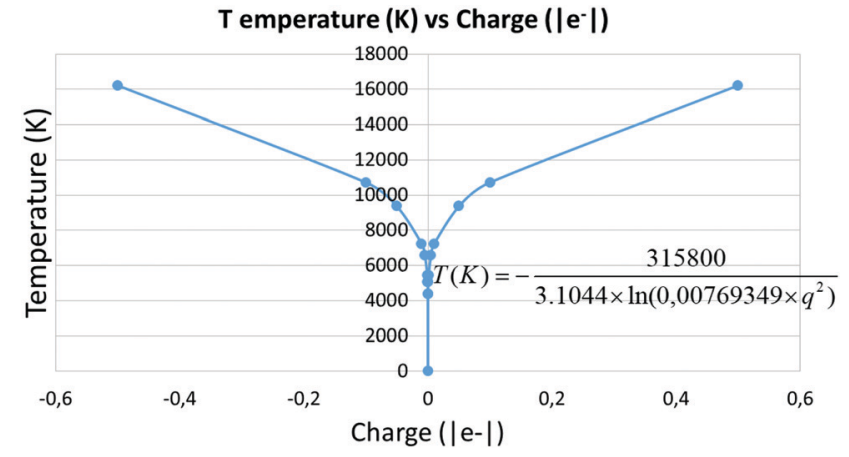

Fig. 5 Temperature as a function of the pertubation charge.

with significant activation barriers. Because of the late character of the transition states of addition, selectivity is assumed to be better grasped through a study of the reaction intermediate, rather than of the starting reagent. From Fig. 6, one may indeed see that addition on the $\alpha$ carbon leads to the best dispersion of the positive charge over the molecule, and thus the associated intermediate (and transition state) should accordingly be the lowest in energy.

However, it is noteworthy that in this particular case the driving force beneath the stabilisation of the reaction intermediate already manifests itself in the starting reagent. Indeed, the higher propensity to disperse the excess positive charge in the $\alpha$ intermediate can be traced back to the higher polarisability of this atom in the heterocycle. As such, we may expect to retrieve the regioselectivity of the electrophilic substitution on these heterocycles from a study of the polarisability of the carbon atoms on the ring.

Again, to check which atom is the most polarisable or soft, a perturbing positive point charge $(+0.1 e)$ has been successively located on each nucleus. As the molecule is symmetrical, only two carbon positions have been tested, namely the $\alpha$ and $\beta$ carbons. The density polarisation maps are represented in Fig. 7. The color code is unchanged, the electron density depletion regions are colored in yellow, while the regions that gain electrons are in red. Qualitatively, the same maps are obtained for both pyrrole and thiophene. The depletion regions for a perturbation located on the $\alpha$ carbon are the neighbour $\beta$ and opposite $\alpha$ carbons. This is in agreement with the Lewis structures represented in Fig. 6 . The same goes for the perturbation positioned on the nucleus of the $\beta$ carbon. In this case

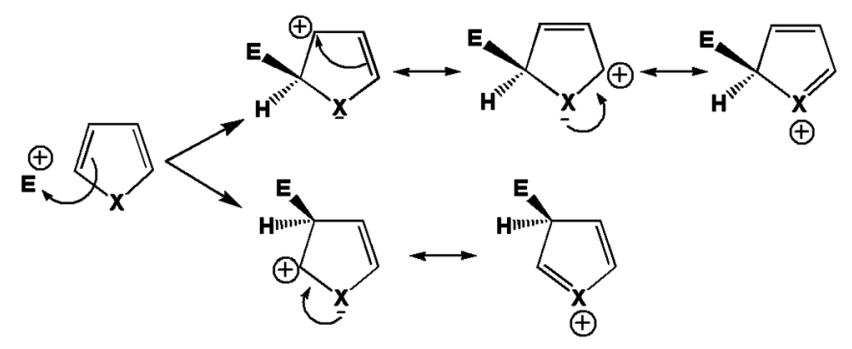

Fig. 6 First step of the aromatic electrophilic attack on a five member ring heterocycle. 

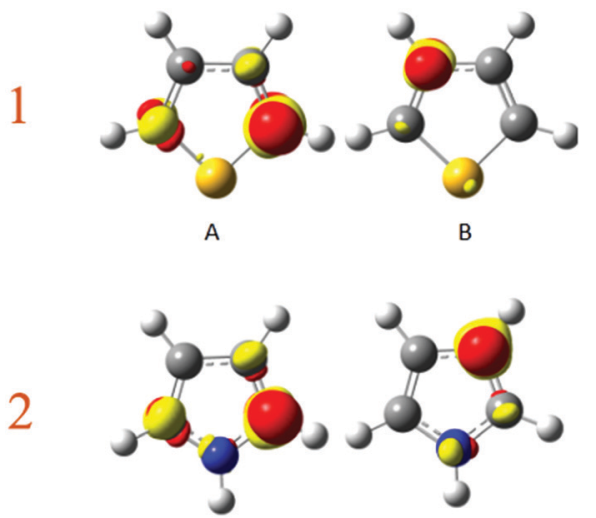

Fig. 7 Density polarisation map for perturbation on carbon (A) $\alpha$ and (B) $\beta$ of thiophene (1) and pyrrole (2). Isovalue of $0.0005 \mathrm{e} \mathrm{bohr}^{-3}$.

the electron density depletion is principally located on the alpha carbon and the heteroatom, once again in full compliance with the Lewis structures in Fig. 6.

It is now important to assess polarisation energy and polarisation entropy for both cases to check whether they are good indicators of the stabilization of the additional charge. The decomposition spectra for both molecules and both perturbation locations are represented in Fig. 8 and 9 .

In the case of thiophene, it is striking how the polarisation spectra are different between $\alpha$ and $\beta$ positions. Very few excited states are triggered in the former, while many are in the latter. The intensities are also quite different. Indeed, the transition probabilities are four times higher in the former than in the latter. The polarisation entropy and polarisation energies follow the same trends as for acrolein: stronger intensities lead to strong entropy and an important stabilization energy. Polarisation entropy and polarisation energy therefore are accordingly remarkable indices to describe the stability of the late reaction intermediate formed by the attack of an electrophile on thiophene.

The same trend is also observed for pyrrole. When the perturbation is located in carbon $\alpha$, fewer excited states are triggered than that of carbon $\beta$. The transition probabilities are nevertheless bigger for the former than for the latter leading to a larger polarisation entropy and a stronger polarisation energy

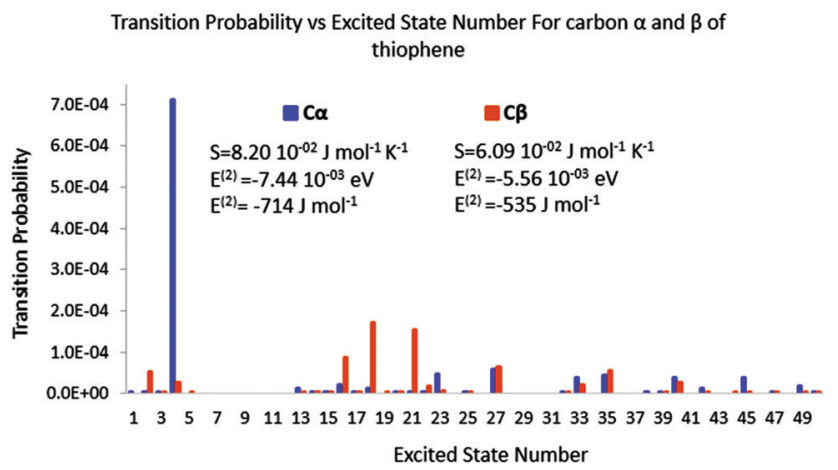

Fig. 8 Polarisation spectrum when $C \alpha$ or $C \beta$ of thiophene is perturbed by a 0.1 e point charge.

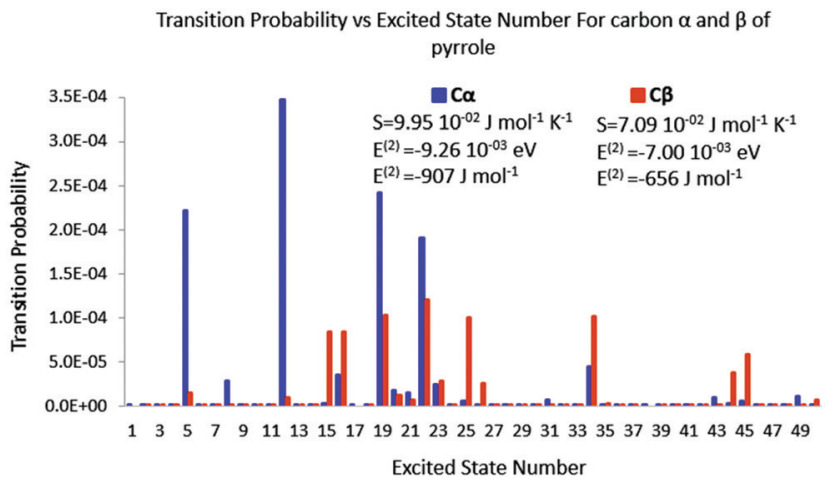

Fig. 9 Polarisation spectrum when $C \alpha$ or $C \beta$ of pyrrole is perturbed by a -0.1 e point charge.

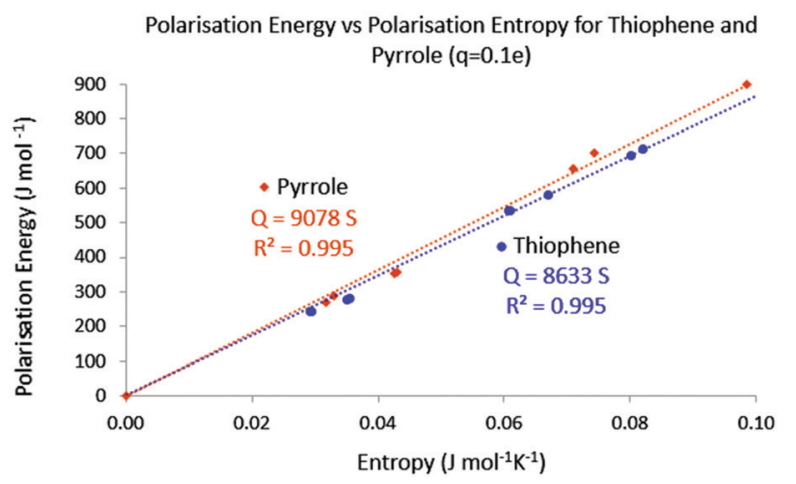

Fig. 10 Absolute polarisation energy with respect to the polarisation entropy for thiophene and pyrrole. $q=0.1 \mathrm{e}$

stabilisation. Again the polarisation energy and the polarisation entropy are good descriptors of the stability of the late reaction intermediate.

Finally the temperature has been calculated using the same protocol. In Fig. 10 the polarisation energy with respect to the polarisation entropy for the perturbation point charge located successively on each nucleus is represented. Just like for acrolein, a linear relationship connects the polarisation energy to the polarisation entropy, with a temperature close to $8600 \mathrm{~K}$. This procedure done on pyrrole leads to a temperature of $9100 \mathrm{~K}$. Again these ranges of temperature are in agreement with the calculated temperature needed to excite 1 percent of the total population of a collection of molecules in the first excited state.

\section{Concluding remarks}

The main goal of this paper was to show that a close analogy can be drawn between conceptual DFT and statistical thermodynamics. This analogy has led to the proposal of a new perspective on work, heat, entropy and also temperature associated with the electron system. It must be noted that their calculations come with quite a heavy price. Indeed, lots of excited states must be computed. An assessment of their added 
values with respect to more traditional DFT descriptors has to be undertaken. However, they thus complement the standard temperature defined and used in molecular dynamics, which is related to kinetic energy of the nuclei, and thus to the nuclear moves. In this contribution, we gave compelling evidence that the application of this concept to the electron density provides useful hints on chemical reactivity. More precisely, when an isolated molecule undergoes a perturbation of the potential it is submitted to, the electronic energy variation can be seen in terms of electronic work and electronic heat exchange, the latter being invoked when an electron density polarisation is induced.

This density reshuffling can be further approached as a cooling (heat exchange) and is translated by a modification of the energy level occupations. It follows that an electron distribution entropy can be defined along with an electronic temperature. The polarisation entropy thus defined is able to characterize the stabilization due to the reshuffling and can be used to predict the stability of a chemical reaction intermediate. Many articles have recently focused upon the variation of DFT descriptors with temperature. In many of them, this latter concept remains elusive. It is believed that this approach may pave the way of the definition of temperature-dependent conceptual DFT descriptors.

\section{Conflicts of interest}

There are no conflicts to declare.

\section{Acknowledgements}

The authors gratefully acknowledge the GENCI/CINES (Project cpt2130) for HPC resources/computer time and the LABEX SynOrg (ANR-11-2ABX-0029) for funding.

\section{Notes and references}

1 S. K. Ghosh, M. Berkowitz and R. G. Parr, Proc. Natl. Acad. Sci. U. S. A., 1984, 81, 8028-8031.

2 P. Hohenberg and W. Kohn, Phys. Rev., 1964, 136, B864.

3 J. F. Capitani, R. F. Nalewajski and R. G. Parr, J. Chem. Phys., 1982, 76, 568.

4 R. G. Parr, R. A. Donnelly, M. Levy and W. E. Palke, J. Chem. Phys., 1978, 69, 3801.

5 L. J. Bartoletti and R. G. Parr, J. Chem. Phys., 1980, 72, 1593-1596.

6 T. Gál and A. Nagy, Mol. Phys., 1997, 91, 873-880.

7 A. Nagy, R. G. Parr and S. Liu, Phys. Rev. A: At., Mol., Opt. Phys., 1996, 53, 3117-3121.

8 P. Ayers, R. Parr and A. Nagy, Int. J. Quantum Chem., 2002, 90, 309-326.

9 R. G. Parr, K. Rupnik and S. K. Ghosh, Phys. Rev. Lett., 1986, 56, 1555-1558.

10 P. K. Chattaraj and S. Sengupta, J. Phys. Chem. A, 1997, 101, 7893-7900.
11 S. Liu and R. Parr, J. Chem. Phys., 1997, 106, 5578-5586.

12 M. Franco-Pérez, C. A. Polanco-Ramirez, J. L. Gazquez, P. W. Ayers and A. Vela, Theor. Chem. Acc., 2020, 139, 44.

13 M. Franco-Pérez, J. Chem. Phys., 2019, 151, 074105.

14 J. L. Gazquez, M. Franco-Pérez, P. W. Ayers and A. Vela, Int. J. Quantum Chem., 2019, 119, e25797.

15 R. A. Miranda-Quintana, M. Franco-Perez, J. L. Gazquez, P. W. Ayers and A. Vela, J. Chem. Phys., 2018, 149, 124110.

16 M. Franco-Pérez, J. L. Gázquez, P. W. Ayers and A. Vela, J. Phys. Chem. A, 2020, 124(26), 5465-5473.

17 P. Geerlings, E. Chamorro, P. K. Chattaraj, F. De Proft, J. L. Gazquez, S. Liu, C. Morell, A. Toro-Labbe, A. Vela and P. Ayers, Theoretical Chemical Accounts, 2020, 139, 36.

18 P. Geerlings, F. De Proft and W. Langenaeker, Chem. Rev., 2003, 103, 1793-1873.

19 H. Chermette, J. Comput. Chem., 1999, 20, 129-154.

20 J.-D. Chai, J. Chem. Phys., 2012, 136, 154104.

21 J.-D. Chai, J. Chem. Phys., 2014, 140, 18 A521.

22 S. Grimme and A. Hansen, Angew. Chem., Int. Ed., 2015, 54, 12308-12313.

23 N. W. Ashcroft and N. D. Mermin, Solid State Physics, Saunders College, 1976.

24 S. Wilke, M. H. Cohen and M. Scheffler, Phys. Rev. Lett., 1996, 77, 1560-1563.

25 C. Rong, T. Lu, P. K. Chattaraj and S. Liu, Indian J. Chem., 2014, 53A, 970-977.

26 S. Liu, J. Chem. Phys., 2007, 126, 191107.

27 S. Liu, C. Rong and T. Lu, J. Phys. Chem. A, 2014, 118, 3698-3704.

28 W. Wu, Z. Wu, C. Rong, T. Lu, Y. Huang and S. Liu, J. Phys. Chem. A, 2015, 119, 8216-8224.

29 F. Heidar-Zadeh, I. Vinogradov and P. W. Ayers, Theor. Chem. Acc., 2017, 136, 54.

30 F. Heidar-Zadeh, P. W. Ayers, T. Verstraelen, I. Vinogradov, E. Vöhringer-Martinez and P. Bultinck, J. Phys. Chem. A, 2018, 122, 4219-4245.

31 R. F. Nalewajski, Int. J. Mol. Sci., 2002, 3, 237-259.

32 R. F. Nalewajski, Found. Chem., 2014, 16, 27-62.

33 Á. Nagy, Chem. Phys. Lett., 2013, 556, 355-358.

34 J. P. Perdew, R. G. Parr, M. Levy and J. L. Balduz, Phys. Rev. Lett., 1982, 49, 1691-1694.

35 R. G. Parr and W. Yang, Density-Functional Theory of Atoms and Molecules, Oxford University Press, 1989.

36 G. Hoffmann, V. Tognetti and L. Joubert, Chem. Phys. Lett., 2019, 724, 24-28.

37 P. Atkins and J. de Paula, Physical Chemistry, Oxford University Press, 8th edn, 2006.

38 F. Guégan, T. Pigeon, F. De Proft, V. Tognetti, L. Joubert, H. Chermette, P. W. Ayers, D. Luneau and C. Morell, J. Phys. Chem. A, 2020, 124, 633-641.

39 C. E. Shannon, Bell Syst. Tech. J., 1948, 27, 379-423.

40 W. Nernst, Sitzungsberichte der Koniglich Preussischen Akademie der Wissenschaften, 1906, 933-940.

41 Y. Zimmels, Phys. Rev. E: Stat., Nonlinear, Soft Matter Phys., 2002, 65, 036146. 
42 Y. Zimmels, Phys. Rev. E: Stat. Phys., Plasmas, Fluids, Relat. Interdiscip. Top., 1996, 53, 3173-3191.

43 Y. Zimmels, J. Magn. Magn. Mater., 2005, 292, 433-439.

44 F. Neese, Wiley Interdiscip. Rev.: Comput. Mol. Sci., 2012, 2, 73-78.

45 E. Dumont and P. Chaquin, Chem. Phys. Lett., 2007, 435, $354-357$.
46 E. Dumont and P. Chaquin, J. Mol. Struct.: THEOCHEM, 2004, 680, 99-106.

47 R. G. Pearson, J. Am. Chem. Soc., 1963, 85, 3533-3539.

48 F. Carey and R. Sundberg, Advanced Organic Chemistry: Part A: Structure and Mechanisms, Springer US, 2007.

49 G. S. Hammond, J. Am. Chem. Soc., 1955, 77, 334-338. 